Dementia
and Geriatric
Cognitive Disorders
Dement Geriatr Cogn Disord Extra 2013;3:342-350

DOI: $10.1159 / 000354187$

Published onlıne: October 5, 2013 (c) 2013 S. Karger AG, Basel

1664-5464/13/0031-0342\$38.00/0 www.karger.com/dee

This is an Open Access article licensed under the terms of the Creative Commons AttributionNonCommercial 3.0 Unported license (CC BY-NC) (www.karger.com/OA-license), applicable to the online version of the article only. Distribution permitted for non-commercial purposes only.

\title{
Context Memory in Alzheimer's Disease
}

\author{
Mohamad El Haj ${ }^{a-c}$ Roy P.C. Kessels ${ }^{d-f}$ \\ a Laboratoire Epsylon, EA 4556, Université Paul-Valery, Montpellier III, Montpellier, \\ bLaboratoire de Psychologie des Pays de la Loire, L'Université Nantes Angers Le Mans \\ (L'UNAM), Angers, and 'Neuropsychology and Auditory Cognition, Department of \\ Psychology, University of Lille 3, Lille, France; ${ }^{d}$ Donders Institute for Brain, Cognition and \\ Behaviour, Radboud University Nijmegen, Nijmegen, eVincent van Gogh Institute for \\ Psychiatry, Korsakoff Clinic, Venray, and fDepartment of Medical Psychology, Radboud \\ University Nijmegen Medical Centre, Nijmegen, The Netherlands
}

Key Words

Alzheimer's disease · Binding · Context memory · Diagnosis · Episodic memory

\section{Abstract}

Background: Alzheimer's disease (AD) is a neurodegenerative disease characterized by a gradual loss of memory. Specifically, context aspects of memory are impaired in AD. Our review sheds light on the neurocognitive mechanisms of this memory component that forms the core of episodic memory function. Summary: Context recall, an element of episodic memory, refers to remembering the context in which an event has occurred, such as from whom or to whom information has been transmitted. Key Messages: Our review raises crucial questions. For example, (1) which context element is more prone to being forgotten in the disease? (2) How do AD patients fail to bind context features together? (3) May distinctiveness heuristic or decisions based on metacognitive expectations improve context retrieval in these patients? (4) How does cueing at retrieval enhance reinstating of encoding context in AD? By addressing these questions, our work contributes to the understanding of the memory deficits in AD.

(C) 2013 S. Karger AG, Basel

\section{Introduction}

Profound memory deterioration is widely acknowledged as the core symptom of Alzheimer's disease (AD) that is already present in the predementia MCI stage of the disease. In addition, clinicians have identified biological diagnostic markers, although research in AD has 
not yet 'established a firm link between the appearance of any specific biomarker in asymptomatic individuals and the subsequent emergence of clinical symptomatology' [1]. Not surprisingly, a recent revision of AD criteria [2] suggests the need of more research before validating the use of biomarkers in the diagnosis. According to this recommendation, there is little standardization of biomarkers, and access to add-on diagnostic techniques is limited to varying degrees in research settings and clinical centers. These concerns led McKhann et al. [2] to suggest focusing on clinical criteria, providing 'very good diagnostic accuracy and utility in most $[\mathrm{AD}]$ patients'. In line with this suggestion, our paper will try to provide a framework for clinicians and researchers dealing with AD diagnosis, aimed at the underlying cognitive mechanisms of memory failure in these patients. We will do this by analyzing recent neuropsychological findings in research investigating the core symptom of the disease, i.e. memory perturbation. It is noteworthy that memory impairment is considered to be a more accurate predictor of early AD than neurobiological predictors, such as atrophy of the medial temporal lobe [3].

Our paper can be divided into 2 major parts. First, we will establish a state-of-the-art review of the literature investigating context memory in AD. Within this description, the second part of the present work will offer new insights for clinicians evaluating memory decline in $\mathrm{AD}$.

\section{Context Memory Impairment in AD}

According to McKhann et al. [2], memory decline in dementia refers to the impaired ability to acquire and remember new episodic information. This impairment can manifest itself in several symptoms, such as 'repetitive questions or conversations', 'misplacing personal belongings', 'forgetting events or appointments', and 'getting lost on a familiar route'. This definition can be developed by relating each of the mentioned symptoms to a specific context memory function.

\section{Destination Memory in $A D$}

The first symptom of memory decline in $\mathrm{AD}$, as given by McKhann et al. [2], is repetitive questions or conversations. Although this behavior can be intuitively related to forgetting the information per se, recent research has suggested an alternative hypothesis. The repetitive tendency of $\mathrm{AD}$ patients, such as asking the same question several times, may be attributed to forgetting the destination to which the information has been initially outputted (e.g., the person to whom the question has previously been addressed) rather than to forgetting the question per se. Remembering the destination has been found to be particularly deficient in AD patients [4, 5]. El Haj et al. [4] have recently asked AD patients and older control participants to tell facts about pictures of famous people and to remember, in a subsequent recognition task, the destination to which each fact has previously been told. This procedure showed a significant deterioration in the destination recall of the AD patients relative to the older control participants, an outcome that was confirmed by another study [5].

Further evidence which supports attributing the repetitive behavior in AD to forgetting of the destination rather than the information per se comes from studies on normal aging, where people showed less difficulties remembering an item than the context in which the item was acquired [6]. Context recall is likely to be more challenging than item memory because it requires remembering not only the item, but also the conjunction of the item and learning context. From this perspective, the encoding and retrieval of destination information may require integrative processes binding an item and its context destination; this may not be required for item memory. This assumption may explain why destination recall in AD patients 
is more impaired than item recall. Forgetting the destination may also account for the third and fourth symptoms of memory perturbation as described by McKhann et al. [2]. For instance, after getting lost on a familiar route, the first question to be asked is 'where am I heading?'.

In summary, destination recall is found to be particularly affected by $\mathrm{AD}$, a decline that may underlie several symptoms of memory loss, as observed in the disease. Apart from a few studies performed in relation to this memory component, there is an important body of literature investigating source memory in AD patients.

\section{Source Memory in $A D$}

Deteriorating source memory can be related to the second manifestation of memory impairment in $\mathrm{AD}$ patients as described by McKhann et al. [2], i.e. 'misplacing personal belongings'. Source memory can be defined as remembering the episodic context from which information was derived (e.g., 'where have I placed the keys?') [7]. Source memory can be also defined more broadly to include any context aspects that were present when an event occurred, such as perceptual, spatiotemporal, affective, and social elements [7].

Distortions of source memory in AD patients are thought to be related to confabulations [8] and misattributions [9]. Studies show that these patients have difficulties remembering (1) whether memories have previously been acquired via the researcher or via someone else [10], (2) whether words have previously been generated by the researcher or by themselves [11], (3) whether actions have previously been imagined or performed by themselves [12], and (4) whether objects have previously been placed (4a) or have been imagined to have been placed (4b) in a bag by themselves or by the researcher, and whether the researcher has been wearing a black or a white glove when placing objects in a bag (4c) [13].

Many studies so far have demonstrated large source memory deficits in $\mathrm{AD}$, a distortion than can be related to the second manifestation of memory decline in these patients as described by McKhann et al. [2], i.e. 'misplacing personal belongings'.

\section{Context Memory in $A D$}

Both destination and source memory reflect dimensions of context memory. The difference between these dimensions is the direction of information processing, i.e. output versus input. Studies evaluating source memory need to investigate a wide range of different information about the context in which an event occurred, such as when and where it happened, whether it was self- or other-generated, heard or seen, perceived or imagined, or in what voice it was spoken. Such 'extensions' have not yet been defined for destination memory, due to the relatively recent interest in this memory component. However, it is worth noting that El Haj et al. [4] evaluated - as mentioned above - imagined and perceived context destination memory by asking mild AD patients to remember whether they have previously told or imagined telling facts about pictures of famous people. Relative to enacted destination memory, imagined destination memory was found to be particularly bad. However, no significant interaction between group and task condition was detected. What is of interest here is that, similar to source memory, the definition of destination memory can be broadened to various aspects of context memory. Even more interestingly, our data suggest a pronounced decline of context recall in the mild stages of AD. Context memory seems to deteriorate early in $\mathrm{AD}$, and thus its evaluation may provide a sensitive element for detecting early signs of $\mathrm{MCI}$ in these patients.

By providing elements from the encoding stage, context memory is pivotal for episodic recall. In its narrowest definition, episodic memory refers to the conscious recollection of information about the context in which a unique episode took place [14]. Using this definition, context memory can be considered as a core element of episodic memory. The latter system differs, however, from the former in terms of autonoetic reliving or the subjective experience 
induced by retrieving context elements from the encoding stage. The episodic subjective reliving is found to be particularly affected in $\mathrm{AD}$, which is attributed to the dysfunction of the frontal and hippocampal regions affected by this disease [15].

Next, we will highlight several elements that may underlie the deterioration of context memory in $\mathrm{AD}$, such as a failure in binding and distinctiveness heuristic processes.

Binding of Context Information

Several theoretical frameworks $[16,17]$ postulate that accurate memory of context features of an episode depends on a feature-binding process during encoding, connecting the context features to the central event. The individual features of an episode are generally processed in specialized brain regions and have to be bound together to form a coherent episode [18]. Hence, binding separate context features is crucial for the formation of episodic memories [19].

Binding deficits are thought to decrease the discriminability of one event from another during retrieval. In AD patients, several studies have shown an impairment of the binding processes in the verbal [20] and visual short-term memory [4,21]. This corroborates with the literature, which suggests that during the course of the disease, deficits in associative memory are more pronounced than those in nonassociative memory [22].

Binding impairment in $\mathrm{AD}$ can be attributed to the hippocampal damage seen in the disease. There is a great body of evidence supporting the role of the hippocampus in associative learning and memory [23]. Functional neuroimaging studies have also shown that the hippocampus plays a pivotal role in the associative memory decline in healthy older adults [24]. Needless to say, significant atrophy of the hippocampal volume is demonstrated even in preclinical stages of $\mathrm{AD}$, supporting the theory of binding impairment of context information in the disease.

Processing context information in $\mathrm{AD}$ is challenged not only by the deterioration in binding but also by perturbations in distinctiveness heuristic processes.

Context Memory and Distinctiveness Heuristic

The question how context information can be retrieved can be answered by highlighting distinctive heuristic processes. Episodic memories are typically characterized by vivid, specific, perceptual details, leading to retrieval by providing specific keys about the encoding context. When retrieving episodic memories, individuals may adopt a distinctiveness heuristic process [25], i.e. a retrieval-monitoring operation which rejects competitive memories that lack the distinctive features of the expected memories (e.g., 'the animal in the picture cannot be an elephant because it is lacking a trunk').

The distinctiveness heuristic is thought to be challenging when the critical context features are not perceptual but semantic in nature. In their study, Budson et al. [26] asked their participants either to process semantically related words (e.g., candy and sugar) or to process the same words accompanied with a distinctive picture of the item. Unlike older controls without $\mathrm{AD}, \mathrm{AD}$ patients showed difficulties using the distinctiveness heuristic to reduce their false recognition of the previously processed words. Budson et al. [26] attributed this failure to the assumption that the pictures boosted semantic processing, enhancing semantically based false recognition in $\mathrm{AD}$ patients. In order to test this assumption, the same procedures were replied in a second study [27], which used semantically unrelated words. These procedures showed less false recognition in AD patients who studied pictures than in those who studied words only. This study by Budson et al. [27] suggests that AD patients can remember vivid context details using metacognitive strategies based on the distinctiveness heuristic.

Next, we will illustrate another strategy improving context retrieving in AD patients, namely context cueing. 


\section{Cueing Context Memory in AD}

According to the encoding specificity principle [28], acquisition and retrieval conditions must be matched to maximally enhance episodic recall. This principle is used by most episodic memory tasks. For instance, in the episodic test by Grober and Buschke [29], immediate recall is tested by providing semantic cues (e.g., 'what was the tool shown earlier?'), and failure to respond to this cueing is considered to have a greater sensitivity for the diagnosis of dementia [29]. However, other studies have suggested that AD patients can benefit from context cueing to enhance their recall. In a recent study [30], AD patients showed better autobiographical recall after being exposed to self-chosen music than in silence. Not surprisingly, the autobiographical memory referred to events in which the encoding context contained the chosen music, suggesting that reinstating the same cue that was present in the encoding context may enhance autobiographical recall in AD patients.

The latter enhancement in autobiographical recall was confirmed by another study [31], showing that musically cued autobiographical memories in AD patients were more specific, were accompanied by more emotional content, were retrieved faster, and were engaging less executive processes than memories evocated in silence. This improvement was interpreted in terms of involuntary autobiographical recall, referring to a rapid interaction between cue and memory trace that directly activates the hippocampal-neocortical ensemble [32].

The key point here is that the deterioration in context memory in AD patients can be overcome by providing context cues from the encoding phase. Cognitive-psychological [28] and mathematical [33] models of memory postulate that remembering depends on the overlapping between encoding and retrieval stages, with a better performance predicted, the greater the match between both stages is.

\section{Conclusion}

All studies mentioned so far suggest a profound impairment of context memory in AD. AD patients seem to have particular difficulties remembering the destination and the source of processed information, accounting for the symptomatology of their memory disturbances. More specifically, destination perturbation can explain why these patients get lost on a familiar route. A decline of source memory can explain why they tend to forget where objects are placed.

\section{Future Prospects in Evaluating Memory Decline in AD}

\section{Evaluating Context Memory}

Despite the deterioration of context memory in AD, little research has been done in this field so far. There is a need for research which simultaneously evaluates several context features within the same population (e.g., when, where, how, from whom has information been received, and to whom has it been delivered). These research questions are of crucial interest for specifying which context aspect is more prone to being forgotten in AD. Considering episodic reliving, it is also of interest to investigate whether the specific decline of context memory can predict deterioration in autonoetic consciousness, as observed in the disease. Research tends to suggest a relationship between general memory performance and autonoetic consciousness in neurodegenerative diseases [34].

Further studies are also required to clarify processes related to the decline of context memory in $\mathrm{AD}$, such as failures in binding at encoding and perturbations in distinctiveness heuristic of retrieval (see also below). There is also a need to evaluate how cueing at retrieval may enhance context and, consequently, episodic recall in AD. In order to illustrate our purpose, the relationships between context memory, episodic recall, binding, distinctiveness heuristic, and cueing at retrieval are illustrated in figure 1. 


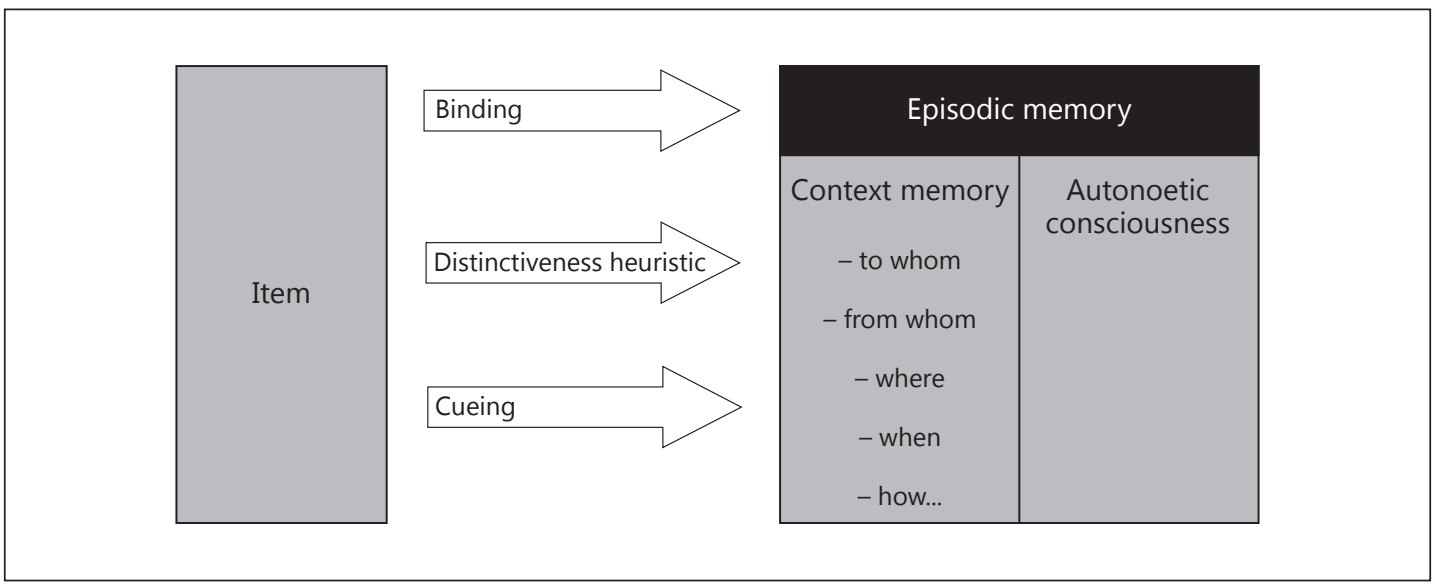

Fig. 1. Future studies should assess (1) the binding of items to context memory during encoding and (2) the distinctiveness heuristic and (3) the cueing of context during retrieval. Further research should also investigate which context aspects are more prone to forgetting in $\mathrm{AD}$ patients and how such declines predict deterioration in autonoetic consciousness.

\section{Evaluating Binding}

A prerequisite for understanding the decline in context memory in AD is the identification of mechanisms preventing the binding of information to its corresponding context. As mentioned earlier, $\mathrm{AD}$ patients show an impairment of the binding processes in their verbal [20] and visual short-term memory [4, 21]. Although they are possibly related to the hippocampal deterioration in $\mathrm{AD}$, the cognitive mechanisms underlying this decline are still not clearly understood. According to Chalfonte and Johnson [35], there are 2 types of bindings in memory. In the first type, features may remain separately represented but are associated. In the second type, features may be associated to form a new representation. The latter type corresponds to the idea that the separate features of information may be less available, when they belong to a newly formed representation. Regarding AD, it is of interest to consider both assumptions about binding, i.e. to evaluate whether $\mathrm{AD}$ patients have difficulties (1) associating features and/or (2) attributing new signification to features when they are bound together.

As AD is mainly associated with medial temporal lobe atrophy, it would be of interest to highlight its involvement in binding processes. Patients with hippocampal lesions show deficits in the short-term maintenance of associations between information [36], and neuroimaging studies show a hippocampal activation during memory binding [16, 19, 37]. In a study by Piekema et al. [19], more medial temporal lobe activation was detected in a spatial binding condition requesting that a number and its location are associated than in a nonspatial binding condition requesting that a number and its color are associated. Considering these outcomes, we wonder whether nonspatial binding would be more efficient in AD patients than spatial binding. It is noteworthy that studies investigating binding in AD patients have assessed either spatial associations (i.e. item-location) [e.g. 4] or nonspatial associations (i.e. item-color) [e.g. 21], urging the need for assessing both binding processes in these patients.

Another fundamental binding process that must be investigated in AD is reactivation. Reactivation, a process by which information is refreshed, is thought to bind information to a new active representation [35]. Another way in which reactivation modulates binding is that repetition increases the strength between already associated features [35]. In this view, reactivation serves a double role, i.e. promoting new binding and strengthening existing asso- 
ciations. Keeping this in mind, future research should investigate whether reactivation could overcome binding deficits in AD patients. In general, studies show beneficial effects of rehearsal strategies in $\mathrm{AD}$ patients [38].

In summary, investigating the decline of context binding is essential because such an impairment may not only affect memory in terms of the association of features, but also memory of the features themselves. Understanding binding deficits may also shed light on the deterioration of episodic encoding in AD. The literature suggests that binding processes occur during the first stage of learning [35] and that memory deficits in AD result from a binding deficit at the initial stage of episodic memory formation [39].

\section{Improving Distinctiveness Heuristic}

AD patients may benefit from distinctiveness heuristic processes when evaluating context features, especially when the critical features are semantically unrelated [27]. Considering this, distinctiveness heuristic processing in these patients may be enhanced by providing distinctive items that can easily be differentiated from related lures. For instance in everyday life, a red landline phone can easily be detected among several black-colored technological tools.

Another strategy enhancing rejection of semantically related context lures is the recallto-reject strategy [40]. Here, lures are rejected by processing logically inconsistent information (e.g., 'this tool is arguably not a landline phone because it does not have a speaker'). Although research suggests recall-to-reject failures in AD patients [41], there is, to the best of our knowledge, no published paper evaluating the logical processes underlying this strategy in these patients.

\section{Cueing Context Memory}

Cueing autobiographical recall in $\mathrm{AD}$ patients with music shows outstanding results [30, 31]. However, there are several questions that need to be answered before we fully understand the benefits of such a phenomenon. For instance, can other sensory-perceptual cues (e.g., odors) enhance recall in these patients as much as music does? One study [42] found that young adults who were presented with odor at both learning and retrieval of a word list showed a better memory than controls receiving odor at just one of these occasions. The validity of these findings remains to be studied in $\mathrm{AD}$ patients.

The neural bases of context-cued memories in AD patients are also worth investigating. To date, it is still unclear how involuntary memories, like those triggered by music [30,31], are neurally activated. Positron emission tomography scans show a larger activation in the right dorsolateral prefrontal cortex (Brodmann areas 8 and 9) and the left precuneus (Brodmann area 7) during voluntary than involuntary recall, while involuntary recall is characterized by an activation in the left dorsolateral prefrontal cortex (Brodmann area 9) [43]. The latter finding corroborates with a study showing an activation in the dorsal regions of the medial prefrontal cortex through a music-evoked autobiographical recall in young adults [44]. Future studies should investigate whether AD patients show the same activation when cueing context recall. It could be that such an activation compensates for the hippocampal dysfunction in these patients.

\section{Concluding Comments}

Our review focuses on the cognitive mechanisms underlying memory decline in AD. Using the symptomatology as described by McKhann et al. [2], we illustrate the importance of evaluating context memory when diagnosing $\mathrm{AD}$. As the results of the reviewed studies 
El Haj et al.: Context Memory in Alzheimer's Disease

show, there is a great need to evaluate context memory when dealing with the diagnosis of AD. These patients seem to have particular difficulties remembering the context in which an event was processed, which affects their episodic phenomenological reliving. Surprisingly, so far no standardized test has been developed to evaluate the decline of context memory in AD patients. Researchers and clinicians should take this into consideration and try to create reliable tests.

Investigating the deterioration of context memory in $\mathrm{AD}$ patients provides insights into memory perturbation at the preclinical stage of the disease, redefining the boundaries between symptomatic and asymptomatic phases. Finally, as Thomson and Tulving [28] suggest, cueing retrieval may dramatically influence remembering by facilitating or blocking the reinstatement of encoding context. Could a rehabilitation program focusing on this assumption improve memory recall in $\mathrm{AD}$ patients?

\section{Disclosure Statement}

The authors report no conflicts of interest. This paper was supported by a VIDI Innovational Research grant from the Netherlands Organization for Scientific Research (NWO MAGW 452-08-005), awarded to R.P.C.K.

\section{References}

1 Sperling RA, Aisen PS, Beckett LA, Bennett DA, Craft S, Fagan AM, et al: Toward defining the preclinical stages of Alzheimer's disease: recommendations from the National Institute on Aging-Alzheimer's Association workgroups on diagnostic guidelines for Alzheimer's disease. Alzheimers Dement 2011;7:280-292.

-2 McKhann GM, Knopman DS, Chertkow H, Hyman BT, Jack CR Jr, Kawas CH, et al: The diagnosis of dementia due to Alzheimer's disease: recommendations from the National Institute on Aging-Alzheimer's Association workgroups on diagnostic guidelines for Alzheimer's disease. Alzheimers Dement 2011;7:263-269.

-3 Schmand B, Huizenga HM, van Gool WA: Meta-analysis of CSF and MRI biomarkers for detecting preclinical Alzheimer's disease. Psychol Med 2010;40:135-145.

4 El Haj M, Postal V, Allain P: Destination memory in Alzheimer's disease: when I imagine telling Ronald Reagan about Paris. Cortex 2013;49:82-89.

5 El Haj M, Postal V, Le Gall D, Allain P: Destination memory in mild Alzheimer's disease. Behav Neurol 2013;26: 215-216.

6 Glisky EL, Polster MR, Routhieaux BC: Double dissociation between item and source memory. Neuropsychology 1995;9:229-235.

7 Johnson MK, Hashtroudi S, Lindsay DS: Source monitoring. Psychol Bull 1993;114:3-28.

-8 Barba GD, Nedjam Z, Dubois B: Confabulation, executive functions, and source memory in Alzheimer's disease. Cogn Neuropsychol 1999;16:385-398.

\9 Mitchell JP, Sullivan AL, Schacter DL, Budson AE: Misattribution errors in Alzheimer's disease: the illusory truth effect. Neuropsychology 2006;20:185-192.

10 Goldman WP, Winograd E, Goldstein FC, O'Jile J, Green RC: Source memory in mild to moderate Alzheimer's disease. J Clin Exp Neuropsychol 1994;16:105-116.

11 Multhaup KS, Balota DA: Generation effects and source memory in healthy older adults and in adults with dementia of the Alzheimer type. Neuropsychology 1997;11:382-391.

12 Fairfield B, Mammarella N: The role of cognitive operations in reality monitoring: a study with healthy older adults and Alzheimer's-type dementia. J Gen Psychol 2009;136:21-39.

13 El Haj M, Fasotti L, Allain P: Source monitoring in Alzheimer's disease. Brain Cogn 2012;80:185-191.

14 Tulving E: Episodic memory: from mind to brain. Annu Rev Psychol 2002;53:1-25.

15 Rauchs G, Piolino P, Mézenge F, et al: Autonoetic consciousness in Alzheimer's disease: neuropsychological and PET findings using an episodic learning and recognition task. Neurobiol Aging 2007;28:1410-1420.

16 Mitchell KJ, Johnson MK, Raye CL, D’Esposito M: fMRI evidence of age-related hippocampal dysfunction in feature binding in working memory. Brain Res Cogn Brain Res 2000;10:197-206.

17 Moscovitch M, Umilta C: Conscious and unconscious aspects of memory; in Lister RG, Weingartner JH (eds): Perspectives on Cognitive Neuroscience. New York, Oxford University Press, 1991, pp 229-266.

18 Eichenbaum H, Otto T, Cohen NJ: Two component functions of the hippocampal memory system. Behav Brain Sci 1994;17:449-517. 
El Haj et al.: Context Memory in Alzheimer's Disease

19 Piekema C, Rijpkema M, Fernandez G, Kessels RP: Dissociating the neural correlates of intra-item and interitem working-memory binding. PLoS One 2010;5:e10214.

20 Parra MA, Abrahams S, Fabi K, Logie R, Luzzi S, Della Sala S: Short-term memory binding deficits in Alzheimer's disease. Brain 2009;132:1057-1066.

21 Parra MA, Abrahams S, Logie RH, Mendez LG, Lopera F, Della Sala S: Visual short-term memory binding deficits in familial Alzheimer's disease. Brain 2010;133:2702-2713.

22 Lowndes G, Savage G: Early detection of memory impairment in Alzheimer's disease: a neurocognitive perspective on assessment. Neuropsychol Rev 2007;17:193-202.

23 Mayes A, Montaldi D, Migo E: Associative memory and the medial temporal lobes. Trends Cogn Sci 2007;11: 126-135.

-24 Yonelinas AP, Hopfinger JB, Buonocore MH, Kroll NE, Baynes K: Hippocampal, parahippocampal and occipitaltemporal contributions to associative and item recognition memory: an fMRI study. Neuroreport 2001;12: 359-363.

-25 Israel L, Schacter DL: Pictorial encoding reduces false recognition of semantic associates. Psychon Bull Rev 1997;4:577-581.

-26 Budson AE, Sitarski J, Daffner KR, Schacter DL: False recognition of pictures versus words in Alzheimer's disease: the distinctiveness heuristic. Neuropsychology 2002;16:163-173.

27 Budson AE, Dodson CS, Daffner KR, Schacter DL: Metacognition and false recognition in Alzheimer's disease: further exploration of the distinctiveness heuristic. Neuropsychology 2005;19:253-258.

-28 Thomson DM, Tulving E: Associative encoding and retrieval: weak and strong cues. J Exp Psychol 1970;86: 255-262.

29 Grober E, Buschke H: Genuine memory deficits in dementia. Dev Neuropsychol 1987;3:13-36.

30 El Haj M, Postal V, Allain P: Music enhances autobiographical memory in mild Alzheimer's disease. Educ Gerontol 2012;38:30-41.

-31 El Haj M, Fasotti L, Allain P: The involuntary nature of music-evoked autobiographical memories in Alzheimer's disease. Conscious Cogn 2012;21:238-246.

32 Moscovitch M: Memory and working with memory: evaluation of a component process model and comparisons with other models; in Schacter DL, Tulving E (eds): Memory Systems. Cambridge, MIT Press, 1994, pp 269-309.

-33 Hintzman D: Judgments of frequency and recognition memory in a multiple-trace memory model. Psychol Rev 1988;95:528-551.

34 Piolino P, Desgranges B, Belliard S, et al: Autobiographical memory and autonoetic consciousness: triple dissociation in neurodegenerative diseases. Brain 2003;126:2203-2219.

35 Chalfonte BL, Johnson MK: Feature memory and binding in young and older adults. Mem Cognit 1996;24: 403-416.

-36 Olson IR, Page K, Moore KS, Chatterjee A, Verfaellie M: Working memory for conjunctions relies on the medial temporal lobe. J Neurosci 2006;26:4596-4601.

37 Piekema C, Kessels RP, Mars RB, Petersson KM, Fernandez G: The right hippocampus participates in shortterm memory maintenance of object-location associations. Neuroimage 2006;33:374-382.

-38 Souchay C, Moulin CJ, Isingrini M, Conway MA: Rehearsal strategy use in Alzheimer's disease. Cogn Neuropsychol 2008;25:783-797.

39 Quinette P, Guillery-Girard B, Noel A, et al: The relationship between working memory and episodic memory disorders in transient global amnesia. Neuropsychologia 2006;44:2508-2519.

40 Gallo DA, Bell DM, Beier JS, Schacter DL: Two types of recollection-based monitoring in younger and older adults: recall-to-reject and the distinctiveness heuristic. Memory 2006;14:730-741.

-41 Pierce BH, Waring JD, Schacter DL, Budson AE: Effects of distinctive encoding on source-based false recognition: further examination of recall-to-reject processes in aging and Alzheimer disease. Cogn Behav Neurol 2008;21:179-186.

42 Schab FR: Odors and the remembrance of things past. J Exp Psychol Learn Mem Cogn 1990;16:648.

-43 Hall NM, Gjedde A, Kupers R: Neural mechanisms of voluntary and involuntary recall: a PET study. Behav Brain Res 2008; 186:261-272.

44 Janata P: The neural architecture of music-evoked autobiographical memories. Cereb Cortex 2009;19:25792594. 